\title{
Fact and fallacy in measurement of conduction velocity in motor nerves
}

\author{
JOHN A. SIMPSON \\ From the Neurological Unit, Department of Medicine, University of Edinburgh, and Northern \\ General Hospital, Edinburgh
}

It is necessary to examine the possible fallacies before one can accept any statement as 'fact'. This paper will attempt to assess the importance of the more obvious fallacies inherent in the method of measurement of conduction velocity as determined by length and latency measurements and will not take account of possible biological variables such as age of the subject. Only the method of computation by length and latency differences between two stimulated points on a nerve will be considered, as the latency of muscle response from a single peripheral stimulus includes unknown variables, including slowing due to terminal branching, conduction by non-myelinated terminals (at a rate of the order of $0.3 \mathrm{~m}$. $/ \mathrm{sec}$. according to Katz and Miledi, 1963), junctional transmission, and propagation from motor end plates to the recording electrodes. Comparisons of latency may usefully be discussed, but not velocity (Simpson, 1956). These difficulties are eliminated if the evaluation is limited to a stretch of nerve which can be stimulated at two points, since the region of uncertainty is excluded from the calculations, but certain methodological difficulties remain.

\section{TEMPERATURE}

The conduction velocity of human peripheral nerve varies widely with temperature (Helmholtz and Baxt, 1867, 1870). Henriksen (1956) calculated that the mean alteration in conduction velocity of the human ulnar nerve was $2.4 \mathrm{~m}$./sec. per degree Centigrade change in temperature. In the conditions of clinical examination this could introduce an error of up to $6 \mathrm{~m}$./ $\mathrm{sec}$. Fortunately the error is systematic and can be prevented by suitable precautions. There may, however, be a temperature gradient along the axis of the limb, especially with peripheral wasting. This may account for the common finding that conduction is slower in the distal segment of a limb and it is difficult to apply a correction for this factor.

\section{MEASUREMENT OF LATENCY}

The method of calculation requires accurate computation of the time of stimulation at some point on the nerve and of the response at some distant part,
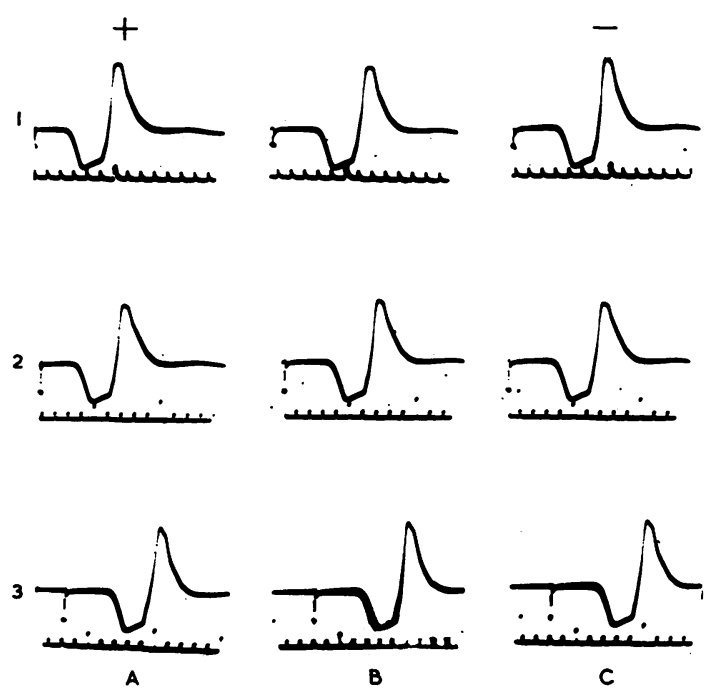

FIG. 1. A Triggering voltage too positive.

$B$ Correct triggering voltage.

$C$ Triggering voltage too negative. Note trigger artefact at 1 msec. which may be mistaken for the stimulus artefact.

1 Free-running time scale. Sweep triggered by stimulator.

2 Time scale and sweep both triggered by stimulator.

3 Time scale and sweep triggered by pre-pulse 3 msec. before stimulus.

Errors due to faulty triggering in oscilloscopes with variable trigger or synchronizing polarity and voltage control do not matter if the stimulus is delayed $(3 A, B, C)$. If the stimulus initiates the sweep $(2,3)$ errors are only obvious if the time scale is also triggered $(2 A, C)$. Only $2 B$ is acceptable. 
either of a muscle fibre or of the compound action potential of the nerve. It is well known that the stimulus pulse is distorted by tissue capacitance but this should not introduce a significant error. Undetected difficulties may arise with inferior recording equipment when the oscilloscope sweep is triggered by the stimulator (Fig. 1). Even if there is no triggering delay, the start of the sweep may be blacked out, or the spot may be momentarily deflected in the wrong direction. These defects may obscure the first two milliseconds and will not be detected if the beam is blanked out between sweeps. Fortunately it is likely to be a systematic error which will not affect the difference between two latencies, but could prevent recognition of distal slowing as in the carpal tunnel syndrome. It is preferable that the stimulus should be delayed after the start of the sweep, and the artefact reduced to a small spike with clearly defined onset. If this is not possible the artefact will be more obvious if the time scale is not free-running but is also started by the triggering pulse.

The determination of the response is more difficult. Motor fibres can only be determined in a mixed nerve by using the muscle response as an index, but it is a complex action potential due to the different velocities of conduction of motor fibres of different calibres. The most readily identified motor units are those with the shortest latency and so the most reliable velocity measurements are for the fastest conducting fibres, but the problem that arises is to decide when the response starts. With surface recording the origin of the action potential of the muscle is curved, making it difficult to decide which point to measure. Recording with high gain minimizes the difficulty but does not remove it (Fig. 2). Recording with a needle electrode suitably positioned makes it easier to determine the onset of the recorded response and has the added advantage of localizing the recorded activity to the desired muscle, but it is theoretically possible that the shortest latency units might be too remote from the electrode to be recorded (Fig. 3). This type of measuring error is not systematic, leading to under- or overestimate of true latency, but has a tendency to cause apparent prolongation. The total error of time measurement may amount to $1 \mathrm{msec}$. but is usually less.

There are two other possible errors of timing. If the pickup electrode is not close to the end-plate zone of muscle there will be an added delay not exceeding $0.35 \mathrm{msec}$. due to conduction along muscle fibres (Henriksen, 1956). This is, however, a systematic variation affecting both readings equally and so not affecting the calculated velocity between two points of stimulation. The other difficulty is introduced by a small negative wave which is some-
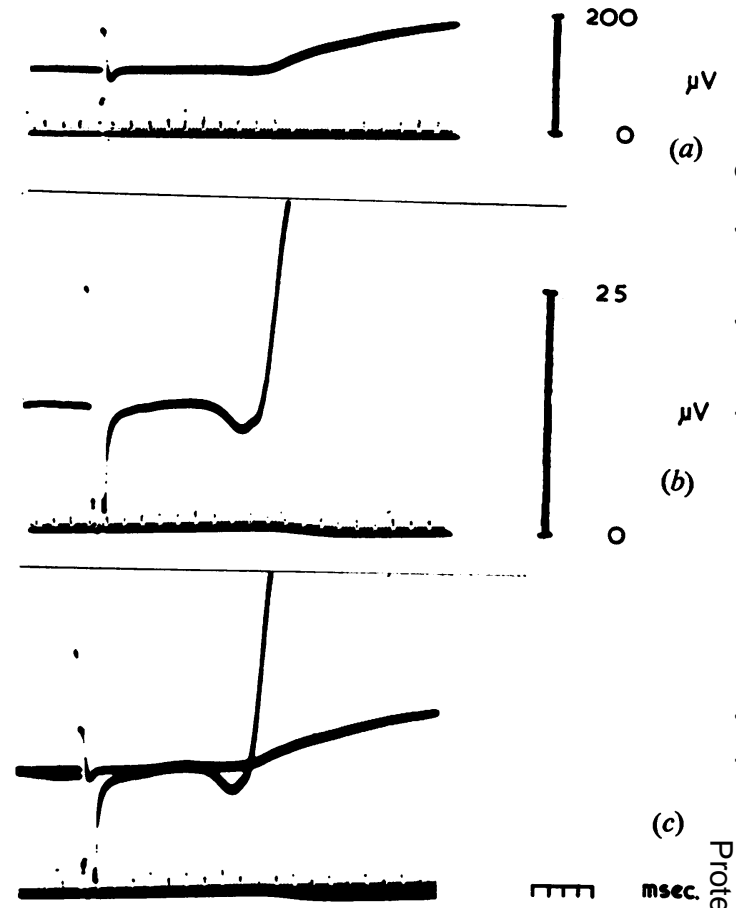

$\mu \mathrm{V}$

(a)

(c)

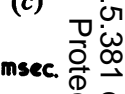

FIG. 2. Stimulus artefact and initial deflection of musct response at amplifier gain (a) suitable for displaying t远 whole of the response, $(b)$ increased $18 d B$ for inspection of initial deflection, (c) both traces superimposed. Surface electrodes.

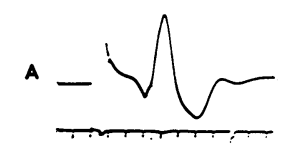

FIG. 3. Evoked response of muscle

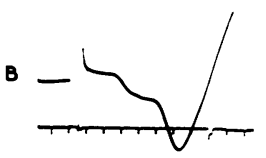
recorded by (a) coaxial needle and (b) surface electrodenear the needle, and (c) on skin $2 \mathrm{~cm}$. away. Each response is superimposed in $(d)$. Time scale in milliseconds.
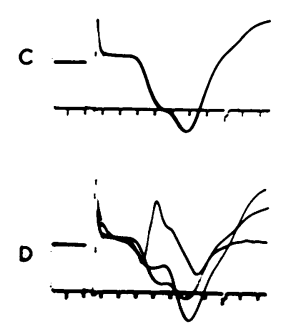

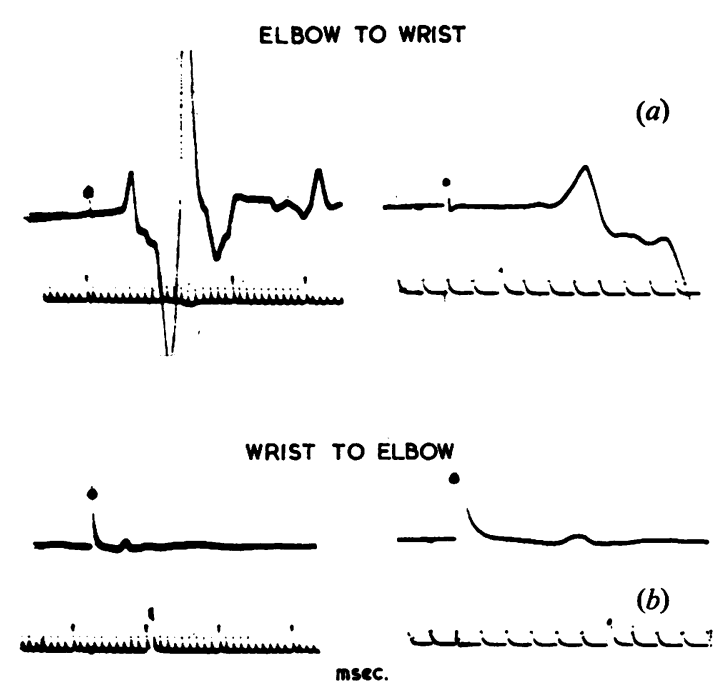

FIG. 4. The initial negative wave of the response recorded from the proximal part of the hypothenar eminence following stimulation of the ulnar nerve at the elbow (a) has the same latency as the action potential recorded from the ulnar nerve at the elbow when the nerve is stimulated at the wrist (b). The initial response in (a) may originate in nerve and not muscle. Time scale in milliseconds (with faster sweep on right).

times recorded immediately before the main muscle complex (Fig. 4). It may be a nerve action potential in the terminal fibres. This is uncertain, but its importance lies in the fact that it is not recorded from all parts of the muscle and may only be seen with high amplification. If the amplifier gain is changed because the muscle potential evoked from one point on the nerve is smaller, the early wave may be taken as the first response to one stimulus and not to the other. Provided it is included in both measurements no difficulty arises in calculating latency difference as this early wave appears to precede the main deflection by a constant amount. It will obviously affect the interpretation when only a single latency measurement is available as in the carpal tunnel syndrome. The nature of this wave requires further investigation.

A possible solution to the difficulty of precise measurement might be to use as a reference point a clearly defined peak of the compound muscle action potential. A moment's consideration will show that this reference point cannot be used. The muscle action potential evoked by indirect stimulation is 10 to $15 \mathrm{msec}$. in duration. The response is not synchronous in every unit because the contributing motor nerve fibres have different conduction velocities. As a result the compound muscle potential is more dispersed when its nerve is stimulated at a distance than when near the muscle (Bolzani, 1954). The difference is less than might be predicted, and Merton (1954) attributes this to artificial synchronization of propagation in different fibres of the muscles by the electrical stimulus. Even with direct recording of the nerve potential, the temporal dispersion is sufficient to make the latency differences significantly greater if measured to the first peak than when measured to the first trough (Dawson, 1956). The position of positive and negative peaks, and indeed the overall duration of the recorded nerve action potential depends on the distance between the recording electrodes. The same principle applies to surface recording of the compound muscle action potential but is minimized by placing the distal electrode over inactive tendon (bellytendon derivation). More precise definition of the onset of the muscular response can be obtained by a suitably placed intramuscular needle electrode, with the additional advantage of greater certainty that the recorded response truly originates in the intended muscle. A possible fallacy is that there may be no motor unit innervated by one of the fastest nerve fibres within the pick-up range of the needle. However, the ability to recognize individual units makes it possible to study the conduction velocity of slower nerve fibres. This may be compromised by the possible muscle synchronization effect referred to above. It is never safe to consider any particular fibre as pathologically slow unless the muscle unit evoked by it fires later than the end of a normal compound action potential. There may simply be a fall-out of the fastest fibres, such as Hodes (1949) suggested occurred in poliomyelitis. This is particularly important with surface recording since it is a common experience that no 'slowing' can be observed until there is a marked fall in the amplitude of the compound muscle potential (Henriksen, 1956). Another difficulty is that nerve fibres may fire iteratively after a single shock stimulus, particularly in compressive neuropathies (Pinelli, 1954; Simpson, 1956). It is often impossible to be certain that the units recorded at the end of a compound muscle potential are not repetitions of units which have already fired with normal latency. This should be suspected if the late unit selected for measurement is separated from the previous unit by the same amount, irrespective of the site of stimulation, or if its latency from one stimulus site shows slight variability.

\section{THE STIMULUS}

If the only reliable units for study are those with the shortest latency, it is most important to ensure that the nerve stimulus is above threshold for all fibres 


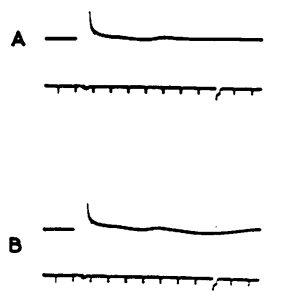

FIG. 5. Effect of increasing intensity of stimulation $(a-d)$. In $(e)$ each is

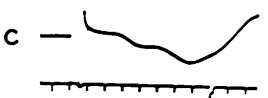
superimposed. Time scale in milliseconds.
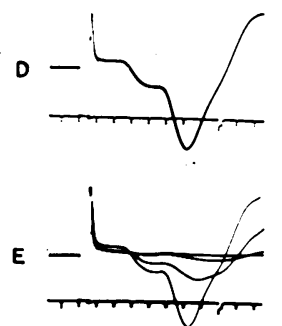

and in practice it should be supramaximal by at least $30 \%$. If the stimulus intensity is gradually increased from below threshold it will be seen that the lowest threshold fibres, though usually rapid, are not always the fastest, since earlier units are recruited by increasing the stimulus (Fig. 5). At near-maximal stimulus intensity there may be seen a continuous decrease in latency and this becomes still shorter even after the muscle action potential has ceased to increase in size (Dawson, 1956). This is probably due to spread of the stimulus from the cathode.

The factors so far considered refer to the identification of a particular motor unit and the determination of its latency. It must be assumed for the purpose of discussion that an accurate time standard is available to be photographed simultaneously with the evoked potential, that the two spots synchronize in sweep speed when separated on the oscilloscope screen, and that accurate means of measuring the photograph are available. When all these factors of identification, recording, and measurement are taken into account, an error of $0.5 \mathrm{msec}$. in each latency measurement is well within the unavoidable error of the method, so that a latency-difference of $1.0 \mathrm{msec}$. might commonly occur. In the conditions of clinical recording it may well be greater.

\section{CONDUCTION DISTANCE}

The uncertainty of the length measurement is still more serious. Unless the stimulating cathode is placed exactly over the nerve (as identified by the point where a muscle response can be evoked with the least stimulus intensity) the measurement must be faulty. Even then there is the possibility that the true cathode is at some distance from the apparen one. Experiments by Henriksen (1956) suggest that this is not an important consideration and that the true cathode may be considered as lying beneath the centre point of a circular cathodal electrode, what ever its size. Nevertheless this need not be true for supramaximal stimulation where the ohmic sprea舟 of current may be sufficient to cause a virtual cathode some distance away, and the shortened latence described above suggests that this does occur. The uncertainty about cathodal position is trivial com $\overrightarrow{\mathrm{D}}$ pared with the fact that it is not possible to make \& direct measurement of the length of nerve stimulatede Uncertainty increases as the nerve assumes an irregular course, especially in the hand or foot.-. It is impossible to measure true velocity in this parf్ of a limb nerve and only comparison of latencies is: possible (Simpson, 1956). Fortunately the maing nerve trunks in the limbs have comparatively straightu courses. Length estimations made by surfacemeasure ${ }^{-1}$ ment must only be approximate. Carpendale (19 $\overline{\not B}) \mathrm{w}$ states that the surface measurement is surprising ${ }^{\infty}$ close to the true length of the nerve between cathodes? provided that the measurement is made with the limb in the same position as it was when stimulated.0 This may be true (it requires confirmation), but की more serious difficulty is the observer error. servers may differ by $1 \mathrm{~cm}$. in measuring the distane between two points on a limb if instructed to lay thes tape along the course of a nerve, especially one such ${ }^{+}$ as the ulnar which winds from dorsal to ventralo surface of the limb.

\section{CALCULATED VELOCITY}

These factors have been investigated by mostō workers in the field including the writer. Henriksen 3 (1956) and Carpendale (1956) concluded that none was significant except the temperature effect and even that would only modify the calculated velocity by about $2 \mathrm{~m}$. $/ \mathrm{sec}$., if reasonable precautions were taken. These conclusions assume that only one factor is operative at a time, but the picture is different if we consider that with reasonable tech- $\delta$ nique there may be a simultaneous and unavoidable

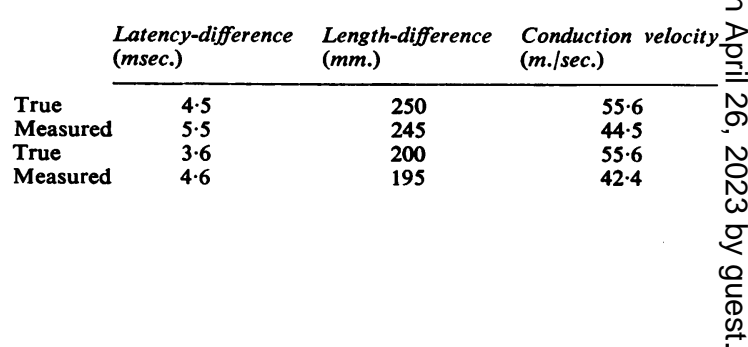


error of $+1.0 \mathrm{msec}$. in latency-difference and $\pm 0.5 \mathrm{~cm}$. in length measurement.

It will be seen from the table that the unavoidable error becomes proportionately greater as the length of nerve is reduced. Fortunately errors acting in the same direction will be comparatively rare; nevertheless Henriksen (1956) found that measurements of the conduction velocity of the ulnar nerve of normal adults repeated on different days could vary by up to $7.5 \mathrm{~m}$./sec. (in 17 cases). In unfavourable conditions the discrepancy could easily be as great as that shown in the table. It may be concluded that no 'borderline' values should be accepted until confirmed by one or more repeat observations with meticulous attention to methodological detail.

\section{ANATOMICAL FACTORS}

Finally it is necessary to point out an important fact which is apparently unfamiliar to many workersthe possibility of 'anastomosis' between peripheral nerves. When investigating the conductivity of the ulnar nerve to abductor digiti minimi one may record a shorter latency when stimulating at the axilla than at the elbow. Also, with cubital lesions of the ulnar nerve, the muscle potential evoked from the elbow is dispersed in time while from the higher point it may be well synchronized. It is possible that the muscle receives double innervation from median and ulnar nerves, since it is rarely possible to be certain that the stimulus has not spread to the median nerve, but such an explanation is very unlikely in view of the large size of the muscle response from axillary stimulation. Murphey, Kirklin, and Finlayson (1946) and Rowntree (1949) showed that double innervation is common in some of the intrinsic muscles of the hand although it is rare in abductor digiti minimi. By blocking the median nerve at the wrist these authors were able to show that fibres passed from the median nerve in the upper part of the forearm to the ulnar nerve to be distributed to the hand muscles. Murphey et al. (1946) demonstrated the anastomoses by dissection in one case and state that they are illustrated in the anatomical textbook by Poirier and Charpy (1899-1907) as single, double, or triple strands crossing from the median to the ulnar nerve between the superficial and deep layers of the flexor muscles of the forearm. If this anastomosis is frequent it would account for the fact that conduction velocity is commonly normal distal to a brachial plexus palsy. This could be due to sparing of fast conducting fibres, as suggested by Trojaborg (1962), but it is more likely to be an example of the fact previously pointed out that conduction may be delayed at the site of a localized lesion and normal distal to it (Simpson, 1956). This should make one cautious about drawing general conclusions about the state of a peripheral nerve from restricted sampling of conduction times. Conduction velocity studies are extremely valuable in clinical medicine, but one must be constantly on guard against the fallacies.

I am glad to acknowledge the assistance of Dr. I. D. Sanderson in the preparation of illustrations.

\section{REFERENCES}

Bolzani, L. (1954). Schweiz. Arch. Neurol. Psychiat., 74, 148. Carpendale, M. T. F. (1956). M.S. (Phys. Med.) Thesis, University of Minnesota.

Dawson, G. D. (1956). J. Physiol. (Lond.), 131, 436.

Helmholtz, H., and Baxt, N. (1867). Mber. K. preuss. Akad. Wiss. Berl., 1867, p. 228.

Henriksen, J. D. (1956). M.S. (Phys. Med.) Thesis, University of Minnesota.

Hodes, R. (1949), J. Neurophysiol., 12, 257.

Katz, B., and Miledi, R. (1963). J. Physiol. (Lond.), 172, 26P

Merton, P. A. (1954). Ibid., 124, 311.

Murphey, F., Kirklin, J. W., and Finlayson, A. I. (1946). Surg. Gynec. Obstet., 83, 15.

Pinelli, P. (1954). Riv. Neurol., 24, 705.

Poirier, P., and Charpy, A. (1899-1907). Traité d'Anatomie Humaine, pp. 1024-1033. Battaile, Paris.

Rowntree, T. (1949). J. Bone Jt Surg., 31B, 505.

Simpson, J. A. (1956). J. Neurol. Neurosurg. Psychiat., 19, 275.

Trojaborg, W. (1962). Dan. med. Bull., 9, 23. 